\title{
Besov spaces on spaces of homogeneous type and fractals
}

\author{
by \\ DACHUN YANG (Beijing)
}

\begin{abstract}
Let $\Gamma$ be a compact $d$-set in $\mathbb{R}^{n}$ with $0<d \leq n$, which includes various kinds of fractals. The author shows that the Besov spaces $B_{p q}^{s}(\Gamma)$ defined by two different and equivalent methods, namely, via traces and quarkonial decompositions in the sense of Triebel are the same spaces as those obtained by regarding $\Gamma$ as a space of homogeneous type when $0<s<1,1<p<\infty$ and $1 \leq q \leq \infty$.
\end{abstract}

1. Introduction. It is well known that the spaces of homogeneous type introduced by Coifman and Weiss in [2] include $\mathbb{R}^{n}$, the surface of the unit ball and the $n$-torus in $\mathbb{R}^{n}$, the $C^{\infty}$ compact Riemannian manifolds, and in particular, the $d$-sets in $\mathbb{R}^{n}$ as special models. It has been proved by Triebel in [15] that these $d$-sets in $\mathbb{R}^{n}$ include various kinds of fractals. Let us first recall the definition of the $d$-sets in the sense of Triebel; see [15, p. 5].

Definition 1.1. Let $n \in \mathbb{N}, \Gamma$ be a set in $\mathbb{R}^{n}$ and $0 \leq d \leq n$. Then $\Gamma$ is said to be a $d$-set if there is a Borel measure $\mu$ in $\mathbb{R}^{n}$ with the following two properties:

(i) $\operatorname{supp} \mu=\Gamma$;

(ii) there are two constants $C>0$ and $C^{\prime}>0$ such that for all $\gamma \in \Gamma$ and all $r$ with $0<r<1$,

$$
C^{\prime} r^{d} \leq \mu(B(\gamma, r) \cap \Gamma) \leq C r^{d} ;
$$

here and in what follows, $B(\gamma, r)=\left\{y \in \mathbb{R}^{n}:|y-\gamma|<r\right\}$.

From this definition, it is easy to see that $\Gamma$ is a closed set in $\mathbb{R}^{n}$, but $\Gamma$ may not be compact. Also, in $[15$, p. 5], Triebel has proved that $\mu$ is actually

2000 Mathematics Subject Classification: Primary 46E35; Secondary 42B35, 43A99.

Key words and phrases: Besov spaces, atoms, blocks, $d$-sets, fractals, spaces of homogeneous type.

The author gratefully acknowledges the support of the Alexander von Humboldt Foundation of Germany, and thanks Professors Hans Triebel and Yongsheng Han for their generously sharing their many ideas with him. The author also thanks the editor for his careful reading of the manuscript and valuable remarks which made this article more readable. This research was partially supported by NNSF (No. 10271015) and RFDP (No. 20020027004) of China. 
a Radon measure, and moreover, $\mu=\mathcal{H}^{d} \mid \Gamma$, where $\mathcal{H}^{d}$ is the $d$-dimensional Hausdorff measure on $\mathbb{R}^{n}$.

Let $\Gamma$ be a compact $d$-set. Triebel has introduced the spaces $B_{p q}^{s}(\Gamma)$ for $s>0$ by use of two different and equivalent methods, namely, traces in [15] and quarkonial decompositions in [16].

The homogeneous Besov and Triebel-Lizorkin spaces on spaces of homogeneous type have been studied in [10]. In [8], the inhomogeneous Besov and Triebel-Lizorkin spaces on spaces of homogeneous type were introduced via the generalized Littlewood-Paley $g$-functions when $p, q \geq 1$. In [9], the inhomogeneous Triebel-Lizorkin spaces were generalized to the cases where $p_{0}<p \leq 1 \leq q<\infty$ via the generalized Littlewood-Paley $S$-functions, where $p_{0}$ is a positive number. In the case of $d$-sets, $p_{0}=1 / 2$.

The main purpose of this paper is to answer a question posed by Triebel in [16, pp. 159-160]. He asked if these spaces $B_{p q}^{s}(\Gamma)$ are the same as those defined by regarding $\Gamma$ as a space of homogeneous type. We will show that the answer is affirmative.

Let us now briefly review the definition of spaces of homogeneous type. A quasi-metric $\varrho$ on a set $X$ is a function $\varrho: X \times X \rightarrow[0, \infty)$ satisfying

(i) $\varrho(x, y)=0$ if and only if $x=y$.

(ii) $\varrho(x, y)=\varrho(y, x)$ for all $x, y \in X$.

(iii) There exists a constant $A \in[1, \infty)$ such that for all $x, y, z \in X$,

$$
\varrho(x, y) \leq A[\varrho(x, z)+\varrho(z, y)] .
$$

Any quasi-metric defines a topology, for which the balls

$$
B(x, r)=\{y \in X: \varrho(y, x)<r\}
$$

for all $x \in X$ and all $r>0$ form a basis.

The following spaces of homogeneous type, studied in [11], are variants of the spaces of homogeneous type introduced by Coifman and Weiss in [2]. In what follows, we let

$$
\operatorname{diam} X=\sup \{\varrho(x, y): x, y \in X\},
$$

and $A \sim B$ means that there are two constants $C_{1}, C_{2}>0$ independent of the main parameters such that $C_{1}<A / B<C_{2}$.

Definition 1.2. Let $d>0$ and $0<\theta \leq 1$. A space of homogeneous type $(X, \varrho, \mu)_{d, \theta}$ is a set $X$ together with a quasi-metric $\varrho$ and a nonnegative Borel regular measure $\mu$ on $X$ with

$$
\operatorname{supp} \mu=X
$$

such that there exists a constant $C>0$ such that for all $0<r<\operatorname{diam} X$ and all $x, x^{\prime}, y \in X$,

$$
\mu(B(x, r)) \sim r^{d}
$$


and

$$
\left|\varrho(x, y)-\varrho\left(x^{\prime}, y\right)\right| \leq C \varrho\left(x, x^{\prime}\right)^{\theta}\left[\varrho(x, y)+\varrho\left(x^{\prime}, y\right)\right]^{1-\theta}
$$

REMARK 1.1. It is easy to see that if $\operatorname{diam} X<\infty$, then (1.1) holds for all $0<r<\operatorname{diam} X$ if and only if it holds for all $0<r<1$.

Remark 1.2. From (1.1), it is easy to deduce $\mu(\{x\})=0$ for all $x \in X$. This means that the spaces of homogeneous type defined in Definition 1.2 are atomless measure spaces.

If we choose $d=1$ in the above definition, then Macías and Segovia [14] have proved that in the sense of topology equivalence, the spaces $(X, \varrho, \mu)_{d, \theta}$ are just the spaces of homogeneous type in the sense of Coifman and Weiss, whose definition only requires that $\varrho$ is a quasi-metric without (1.2) and $\mu$ satisfies the following doubling condition which is weaker than (1.1): there is a constant $A^{\prime}>0$ such that for all $x \in X$ and all $r>0$,

$$
\mu(B(x, 2 r)) \leq A^{\prime} \mu(B(x, r)) .
$$

However, in [14], Macías and Segovia have shown that for the spaces of homogeneous type in the sense of Coifman and Weiss, one can replace the original quasi-metric $\varrho$ by another quasi-metric $\bar{\varrho}$, which yields the same topology on $X$, such that there exist $C>0$ and $\bar{\theta} \in(0,1]$ satisfying

$$
\bar{\varrho}(x, y) \sim \inf \{\mu(B): B \text { is a ball containing } x \text { and } y\}
$$

and (1.2) with $\varrho$ and $\theta$ replaced, respectively, by $\bar{\varrho}$ and $\bar{\theta}$, and that $\mu$ satisfies (1.1) with $d=1$ for balls corresponding to this new quasi-metric. Moreover, there is a positive constant $C_{0}$ such that $\varrho(x, y)^{1 / C_{0}}$ is equivalent to a metric on $X \times X$. It is easy to see that the set $X$ with this new quasi-metric $\bar{\varrho}$, the original measure $\mu$ and the balls corresponding to the new quasi-metric satisfies (1.1) with $d=1$ and (1.2).

The above variant definition of spaces of homogeneous type is convenient. In fact, $\left(\mathbb{R}^{n}, \varrho, m\right)_{n, 1}$ is just the usual $\mathbb{R}^{n}$, where $\varrho$ is the standard Euclidean metric and $m$ is the $n$-dimensional Hausdorff measure, or equivalently, the $n$-dimensional Lebesgue measure. Moreover, any bounded $d$-set $\Gamma$ in $\mathbb{R}^{n}$ with $0 \leq d \leq n$ is just the space $(\Gamma, \varrho, \mu)_{d, 1}$, where $\varrho$ is again the standard Euclidean metric and $\mu$ is a Radon measure on $\Gamma$ with $\operatorname{supp} \mu=\Gamma$. See $[11]$ for more details.

In this paper, we assume that the total measure of $X$ is finite. Also, in what follows, we let

$$
L^{p}(X)=\left\{f: X \rightarrow \mathbb{C} \text { is a } \mu \text {-measurable function and }\|f\|_{L^{p}(X)}<\infty\right\}
$$


for $p \in(0, \infty]$, where

$$
\begin{aligned}
& \|f\|_{L^{p}(X)}=\left\{\int_{X}|f(x)|^{p} d \mu(x)\right\}^{1 / p} \quad \text { for } p \in(0, \infty), \\
& \|f\|_{L^{\infty}(X)}=\underset{x \in X}{\operatorname{ess} \sup }|f(x)| .
\end{aligned}
$$

The organization of this paper is as follows. In the next section, we will recall some related theory on spaces of homogeneous type, which will be used in Section 3. In Section 3, we will establish the connection between the spaces $B_{p q}^{s}(\Gamma)$ defined by trace methods in [15], or equivalently by quarkonial methods in [16], and the Besov spaces $\bar{B}_{p q}^{s}(\Gamma)$ defined by regarding $\Gamma$ as a space of homogeneous type, $(X, \varrho, \mu)_{d, 1}$ with $\varrho(x, y)=|x-y|$ for all $x, y \in \Gamma$ and $\mu=\mathcal{H}^{d}$, the $d$-dimensional Hausdorff measure on $\Gamma$. In fact, we will show that for $0<s<1,1<p<\infty$ and $1 \leq q \leq \infty, \bar{B}_{p q}^{s}(\Gamma)=B_{p q}^{s}(\Gamma)$.

2. Preliminaries. In this section, we recall some known results on spaces of homogeneous type; see also [11]. Let us first recall the definition of the spaces of test functions on $X$ from [10]; see also [7].

Definition 2.1. Fix $\gamma, \beta>0$. A function $f$ defined on $X$ is said to be a test function of type $\left(x_{0}, r, \beta, \gamma\right)$ with $x_{0} \in X$ and $r>0$ if $f$ satisfies the following conditions:

(i) $|f(x)| \leq C \frac{r^{\gamma}}{\left(r+\varrho\left(x, x_{0}\right)\right)^{d+\gamma}}$;

(ii) $|f(x)-f(y)| \leq C\left(\frac{\varrho(x, y)}{r+\varrho\left(x, x_{0}\right)}\right)^{\beta} \frac{r^{\gamma}}{\left(r+\varrho\left(x, x_{0}\right)\right)^{d+\gamma}}$

$$
\text { for } \varrho(x, y) \leq \frac{1}{2 A}\left[r+\varrho\left(x, x_{0}\right)\right] \text {. }
$$

If $f$ is a test function of type $\left(x_{0}, r, \beta, \gamma\right)$, we write $f \in \mathcal{G}\left(x_{0}, r, \beta, \gamma\right)$, and the norm of $f$ in $\mathcal{G}\left(x_{0}, r, \beta, \gamma\right)$ is defined by

$$
\|f\|_{\mathcal{G}\left(x_{0}, r, \beta, \gamma\right)}=\inf \{C:(\mathrm{i}) \text { and (ii) hold }\} .
$$

Here and in what follows, $\theta$ is the same as in (1.2).

Now fix $x_{0} \in X$ and write $\mathcal{G}(\beta, \gamma)=\mathcal{G}\left(x_{0}, 1, \beta, \gamma\right)$. It is easy to see that

$$
\mathcal{G}\left(x_{1}, r, \beta, \gamma\right)=\mathcal{G}(\beta, \gamma)
$$

with equivalent norms for all $x_{1} \in X$ and $r>0$. Furthermore, it is easy to check that $\mathcal{G}(\beta, \gamma)$ is a Banach space. We also define

$$
\mathcal{G}_{0}\left(x_{0}, r, \beta, \gamma\right)=\left\{f \in \mathcal{G}\left(x_{0}, r, \beta, \gamma\right): \int_{X} f(x) d \mu(x)=0\right\},
$$

and the dual space $(\mathcal{G}(\beta, \gamma))^{\prime}$ to be all the continuous linear functionals on $\mathcal{G}(\beta, \gamma)$. We denote by $\langle h, f\rangle$ the natural pairing of elements $h \in(\mathcal{G}(\beta, \gamma))^{\prime}$ 
and $f \in \mathcal{G}(\beta, \gamma)$. It is also easy to see that for all $h \in(\mathcal{G}(\beta, \gamma))^{\prime},\langle h, f\rangle$ is well defined for all $f \in \mathcal{G}\left(x_{0}, r, \beta, \gamma\right)$ with $x_{0} \in X$ and $r>0$.

It is well known that even when $X=\mathbb{R}^{n}, \mathcal{G}\left(\beta_{1}, \gamma\right)$ is not dense in $\mathcal{G}\left(\beta_{2}, \gamma\right)$ if $\beta_{1}>\beta_{2}$, which causes some inconvenience. To overcome this defect, in what follows, we let $\mathcal{G}(\beta, \gamma)$ be the completion of the space $\mathcal{G}(\theta, \theta)$ in $\mathcal{G}(\beta, \gamma)$ when $0<\beta, \gamma<\theta$.

To state the definitions of the inhomogeneous Besov spaces $B_{p q}^{s}(X)$ studied in [8], we need the following approximations to the identity, which were first introduced in [7].

Definition 2.2. A sequence $\left\{S_{k}\right\}_{k \geq 0}$ of linear operators is said to be an approximation to the identity if there exist $\varepsilon \in(0, \theta]$ and $C>0$ such that for all $k \in \mathbb{N} \cup\{0\}$ and all $x, x^{\prime}, y, y^{\prime} \in X$, the kernel $S_{k}(x, y)$ of $S_{k}$ is a function from $X \times X$ into $\mathbb{C}$ satisfying

(i) $S_{k}(x, y)=0$ if $\varrho(x, y) \geq C 2^{-k}$ and $\left\|S_{k}\right\|_{L^{\infty}(X \times X)} \leq C 2^{d k}$;

(ii) $\left|S_{k}(x, y)-S_{k}\left(x^{\prime}, y\right)\right| \leq C 2^{k(d+\varepsilon)} \varrho\left(x, x^{\prime}\right)^{\varepsilon}$;

(iii) $\left|S_{k}(x, y)-S_{k}\left(x, y^{\prime}\right)\right| \leq C 2^{k(d+\varepsilon)} \varrho\left(y, y^{\prime}\right)^{\varepsilon}$;

(iv) $\left|\left[S_{k}(x, y)-S_{k}\left(x, y^{\prime}\right)\right]-\left[S_{k}\left(x^{\prime}, y\right)-S_{k}\left(x^{\prime}, y^{\prime}\right)\right]\right|$ $\leq C 2^{k(d+2 \varepsilon)} \varrho\left(x, x^{\prime}\right)^{\varepsilon} \varrho\left(y, y^{\prime}\right)^{\varepsilon}$

(v) $\int_{X} S_{k}(x, y) d \mu(y)=1$;

(vi) $\int_{X} S_{k}(x, y) d \mu(x)=1$.

Here, $S_{k}(x, y)$ being the kernel of $S_{k}$ means that for suitable functions $f$,

$$
S_{k} f(x)=\int_{X} S_{k}(x, y) f(y) d \mu(y) .
$$

Now, we can introduce the spaces $B_{p q}^{s}(X)$ via the approximations to the identity defined above; these spaces were first studied in [8].

Definition 2.3. Suppose $\varepsilon \in(0, \theta], s \in(-\varepsilon, \varepsilon)$ and $\left\{S_{k}\right\}_{k=0}^{\infty}$ is an approximation to the identity, and let

$$
E_{k}=S_{k}-S_{k-1} \quad \text { for } k \in \mathbb{N}, \quad E_{0}=S_{0} .
$$

The inhomogeneous Besov space $B_{p q}^{s}(X)$ for $1 \leq p, q \leq \infty$ is the collection of $f \in(\stackrel{\mathcal{G}}{ }(\beta, \gamma))^{\prime}$ with $\max (0,-s)<\beta<\varepsilon$ and $0<\gamma<\varepsilon$ such that

$$
\|f\|_{B_{p q}^{s}(X)}=\left\{\sum_{k=0}^{\infty}\left[2^{k s}\left\|E_{k}(f)\right\|_{L^{p}(X)}\right]^{q}\right\}^{1 / q}<\infty .
$$

It was proved in [8] that the above definition is independent of the choices of the approximations to the identity and the pair $(\beta, \gamma)$ with $\max (0,-s)<$ $\beta<\varepsilon$ and $0<\gamma<\varepsilon$. Moreover, by a similar argument, it was proved in [11] that the definition is also independent of the equivalent quasi-metrics satisfying (1.2). 
In [8], atomic decompositions for these spaces were also given. To state them, we need the following construction of Christ [1], which provides an analogue of the grid of Euclidean dyadic cubes on a space of homogeneous type.

Lemma 2.1. Let $(X, \varrho, \mu)_{d, \theta}$ be a space of homogeneous type. Then there exists a collection $\left\{Q_{\alpha}^{k} \subset X: k \in \mathbb{N} \cup\{0\}, \alpha \in M_{k}\right\}$ of open subsets, where $M_{k}$ is some (possibly finite) index set, and constants $\delta \in(0,1), a_{0}>0$ and $C>0$ such that

(i) $\mu\left(X \backslash \bigcup_{\alpha} Q_{\alpha}^{k}\right)=0$ for each fixed $k$ and $Q_{\beta}^{k} \cap Q_{\alpha}^{k}=\emptyset$ if $\alpha \neq \beta$;

(ii) for any $\alpha, \beta, k, l$ with $l \geq k$, either $Q_{\beta}^{l} \subset Q_{\alpha}^{k}$ or $Q_{\beta}^{l} \cap Q_{\alpha}^{k}=\emptyset$;

(iii) for each $(k, \alpha)$ and each $l<k$ there is a unique $\beta$ such that $Q_{\alpha}^{k} \subset Q_{\beta}^{l}$;

(iv) $\operatorname{diam}\left(Q_{\alpha}^{k}\right) \leq C \delta^{k}$;

(v) each $Q_{\alpha}^{k}$ contains some ball $B\left(z_{\alpha}^{k}, a_{0} \delta^{k}\right)$, where $z_{\alpha}^{k} \in X$.

In fact, we can think of $Q_{\alpha}^{k}$ as being essentially a dyadic cube with diameter roughly $\delta^{k}$ and center $z_{\alpha}^{k}$.

The following (dyadic) smooth atoms on a space of homogeneous type were introduced in [10].

Definition 2.4. Fix $\delta \in(0,1)$ and a collection

$$
\left\{Q_{\tau}^{k} \subset X: k \in \mathbb{N} \cup\{0\}, \tau \in M_{k}\right\}
$$

of open subsets satisfying the conditions as in Lemma 2.1. A function $a_{Q_{\tau}^{k}}$ defined on $X$ is said to be a $\gamma$-smooth atom for $Q_{\tau}^{k}$ if

(i) $\operatorname{supp} a_{Q_{\tau}^{k}} \subset B\left(z_{\tau}^{k}, 3 A C \delta^{k}\right)$;

(ii) $\int_{X} a_{Q_{\tau}^{k}}(x) d \mu(x)=0$;

(iii) $\left|a_{Q_{\tau}^{k}}(x)\right| \leq \mu\left(Q_{\tau}^{k}\right)^{-1 / 2}$ and $\left|a_{Q_{\tau}^{k}}(x)-a_{Q_{\tau}^{k}}(y)\right| \leq \mu\left(Q_{\tau}^{k}\right)^{-1 / 2-\gamma / d} \varrho(x, y)^{\gamma}$.

A function $a_{Q_{\tau}^{k}}$ defined on $X$ is said to be a $\gamma$-smooth block for $Q_{\tau}^{k}$ if $a_{Q_{\tau}^{k}}$ satisfies only (i) and (iii) above.

As in the case $X=\mathbb{R}^{n}$ (see [5]), we also define certain inhomogeneous spaces of sequences indexed by "dyadic cubes" $\left\{Q_{\tau}^{k}\right\}_{\tau \in M_{k}, k \in \mathbb{N} \cup\{0\}} \equiv \mathcal{J}$ in $X$, which will characterize the coefficients in the atomic decompositions of $B_{p q}^{s}(X)$. For $-\varepsilon<s<\varepsilon, 1 \leq p, q \leq \infty$, we let $b_{p q}^{s}(X)$ be the collection of all sequences $\lambda=\left\{\lambda_{Q}\right\}_{Q \in \mathcal{J}}$ such that

$$
\|\lambda\|_{b_{p q}^{s}(X)}=\left\{\sum_{k=0}^{\infty}\left[\sum_{\tau \in M_{k}}\left(\mu\left(Q_{\tau}^{k}\right)^{-s / d-1 / 2+1 / p}\left|\lambda_{Q_{\tau}^{k}}\right|\right)^{p}\right]^{q / p}\right\}^{1 / q}
$$

is finite.

We have the following atomic decompositions for the spaces $B_{p q}^{s}(X)$, which were proved in [8]. 
Lemma 2.2. Suppose $-\varepsilon<s<\varepsilon$.

(i) If $1 \leq p, q \leq \infty$ and $f \in B_{p q}^{s}(X) \cap(\mathcal{G}(\beta, \gamma))^{\prime}$ with $0<\beta, \gamma<\varepsilon$, then there exist a sequence $\lambda=\left\{\lambda_{Q_{\tau}^{k}}\right\}_{Q_{\tau}^{k} \in \mathcal{J}} \in b_{p q}^{s}(X)$, $\varepsilon$-smooth atoms $\left\{a_{Q_{\tau}^{k}}\right\}_{k \in \mathbb{N}, \tau \in M_{k}}$ and $\varepsilon$-smooth blocks $\left\{a_{Q_{\tau}^{0}}\right\}_{\tau \in M_{0}}$ such that

$$
f=\sum_{k=0}^{\infty} \sum_{\tau \in M_{k}} \lambda_{Q_{\tau}^{k}} a_{Q_{\tau}^{k}}
$$

with convergence both in the norm of $B_{p q}^{s}(X)$ and in $(\mathcal{G}(\beta, \gamma))^{\prime}$ when $1 \leq$ $p, q<\infty$, and only in $(\mathcal{G}(\beta, \gamma))^{\prime}$ when $1 \leq p, q \leq \max (p, q)=\infty$, and

$$
\|\lambda\|_{b_{p q}^{s}(X)} \leq C\|f\|_{B_{p q}^{s}(X)} .
$$

(ii) Conversely, suppose

$$
f=\sum_{k=0}^{\infty} \sum_{\tau \in M_{k}} \lambda_{Q_{\tau}^{k}} a_{Q_{\tau}^{k}}
$$

in $(\mathcal{G}(\beta, \gamma))^{\prime}$ with $\max (0,-s)<\beta<\varepsilon$ and $0<\gamma<\varepsilon$, where $a_{Q_{\tau}^{0}}$ 's for $\tau \in M_{0}$ are $\varepsilon$-smooth blocks and $a_{Q_{\tau}^{k}}$ 's for $k \in \mathbb{N}$ and $\tau \in M_{k}$ are $\varepsilon$-smooth atoms. Then

$$
\|f\|_{B_{p q}^{s}(X)} \leq C\|\lambda\|_{b_{p q}^{s}(X)}
$$

for $1 \leq p, q \leq \infty$.

Lemma 2.2 was generalized in [11] to the following case when $s>0$.

Lemma 2.3. Suppose $0<s<\varepsilon$.

(i) If $1 \leq p, q \leq \infty$ and $f \in B_{p q}^{s}(X) \cap(\mathcal{G}(\beta, \gamma))^{\prime}$ with $0<\beta, \gamma<\varepsilon$, then there exist a sequence $\lambda=\left\{\lambda_{Q_{\tau}^{k}}\right\}_{Q_{\tau}^{k} \in \mathcal{J}} \in b_{p q}^{s}(X)$ and $\varepsilon$-smooth blocks $\left\{a_{Q_{\tau}^{k}}\right\}_{k \in \mathbb{N} \cup\{0\}, \tau \in M_{k}}$ such that

$$
f=\sum_{k=0}^{\infty} \sum_{\tau \in M_{k}} \lambda_{Q_{\tau}^{k}} a_{Q_{\tau}^{k}}
$$

with convergence both in the norm of $B_{p q}^{s}(X)$ and in $(\mathcal{G}(\beta, \gamma))^{\prime}$ when $1 \leq$ $p, q<\infty$, and only in $(\mathcal{G}(\beta, \gamma))^{\prime}$ when $1 \leq p, q \leq \max (p, q)=\infty$, and

$$
\|\lambda\|_{b_{p q}^{s}(X)} \leq C\|f\|_{B_{p q}^{s}(X)} .
$$

(ii) Conversely, suppose

$$
f=\sum_{k=0}^{\infty} \sum_{\tau \in M_{k}} \lambda_{Q_{\tau}^{k}} a_{Q_{\tau}^{k}}
$$

in $(\mathcal{G}(\beta, \gamma))^{\prime}$ with $0<\beta, \gamma<\varepsilon$, where $a_{Q_{\tau}^{k}}$ for $k \in \mathbb{N} \cup\{0\}$ are $\varepsilon$-smooth blocks. Then

$$
\|f\|_{B_{p q}^{s}(X)} \leq C\|\lambda\|_{b_{p q}^{s}(X)}
$$

for $1 \leq p, q \leq \infty$. 
We also need the following lemma which can be found in $[10$, p. 93]; see also [5].

Lemma 2.4. Let $1 \leq p \leq \infty, \mu, \eta \in \mathbb{N} \cup\{0\}$ with $\eta \leq \mu$ and for "dyadic cubes" $Q_{\tau}^{\mu}$,

$$
\left|f_{Q_{\tau}^{\mu}}(x)\right| \leq\left(1+2^{\eta} \varrho\left(x, z_{\tau}^{\mu}\right)\right)^{-d-\sigma},
$$

where $z_{\tau}^{\mu}$ is the "center" of $Q_{\tau}^{\mu}$ as in Lemma 2.1 and $\sigma>0$ (recall that $\left.\mu\left(Q_{\tau}^{\mu}\right) \approx 2^{-\mu d}\right)$. Then

$$
\left\|\sum_{\tau} \lambda_{Q_{\tau}^{\mu}} f_{Q_{\tau}^{\mu}}\right\|_{L^{p}(X)} \leq C 2^{(\mu-\eta) d} 2^{-\mu d / p}\left(\sum_{\tau}\left|\lambda_{Q_{\tau}^{\mu}}\right|^{p}\right)^{1 / p}
$$

and

$$
\sum_{\tau}\left|\lambda_{Q_{\tau}^{\mu}}\right|\left|f_{Q_{\tau}^{\mu}}(x)\right| \leq C 2^{(\mu-\eta) d} M\left(\sum_{\tau}\left|\lambda_{Q_{\tau}^{\mu}}\right| \chi_{Q_{\tau}^{\mu}}\right)(x),
$$

where $C$ is independent of $x, \mu$ and $\eta$, and $M$ is the Hardy-Littlewood maximal operator on $X$.

3. Relations between function spaces on $d$-sets and spaces of homogeneous type. Let $\Gamma$ be a $d$-set in $\mathbb{R}^{n}$. In this section, we will establish the connection between the spaces $B_{p q}^{s}(\Gamma)$ defined by means of traces or quarks, which were proved to be equivalent in [16] (see also [15]), and the spaces $B_{p q}^{s}(\Gamma)$ defined by regarding $\Gamma$ as a space of homogeneous type (as in Section 2) with the usual Euclidean metric and the $d$-dimensional Hausdorff measure on $\mathbb{R}^{n}$. Let us first recall the definition of the quarks; see [15] and [16].

For $\varepsilon>0$, let $\Gamma_{\varepsilon}=\left\{x \in \mathbb{R}^{n}:\right.$ dist $\left.(x, \Gamma)<\varepsilon\right\}$ be the $\varepsilon$-neighborhood of $\Gamma$. Let $k \in \mathbb{N}_{0}$ and let $M_{k} \in \mathbb{N}$. For $k \in \mathbb{N}_{0}$, let $\left\{\gamma^{k, m}: m=1, \ldots, M_{k}\right\} \subset \Gamma$ and let $\left\{\psi^{k, m}: m=1, \ldots, M_{k}\right\}$ be approximate lattices and subordinate resolutions of unity with the following properties: There are positive numbers $C_{1}, C_{2}$ and $C_{3}$ with

$$
\left|\gamma^{k, m_{1}}-\gamma^{k, m_{2}}\right| \geq C_{1} 2^{-k}, \quad k \in \mathbb{N}_{0}, m_{1} \neq m_{2},
$$

and

$$
\Gamma_{\varepsilon_{k}} \subset \bigcup_{m=1}^{M_{k}} B\left(\gamma^{k, m}, C_{2} 2^{-k}\right)=\bigcup_{m=1}^{M_{k}} B_{k m}, \quad k \in \mathbb{N}_{0},
$$

where $\varepsilon_{k}=C_{3} 2^{-k}$. Furthermore, $\psi^{k, m}$ 's are nonnegative $C^{\infty}\left(\mathbb{R}^{n}\right)$ functions in $\mathbb{R}^{n}$ with $\operatorname{supp} \psi^{k, m} \subset B\left(\gamma^{k, m}, C_{2} 2^{-k+1}\right)$,

$$
\left|D^{\alpha} \psi^{k, m}(x)\right| \leq C_{\alpha} 2^{k|\alpha|}
$$


for $x \in \mathbb{R}^{n}$ and $\alpha=\left(\alpha_{1}, \ldots, \alpha_{n}\right) \in(\mathbb{N} \cup\{0\})^{n}=\mathbb{N}_{0}^{n}$, and

$$
\sum_{m=1}^{M_{k}} \psi^{k, m}(x)=1
$$

for $x \in \Gamma_{\varepsilon_{k}}$, where $C_{\alpha}$ is a constant, $D^{\alpha}=\left(\partial / \partial x_{1}\right)^{\alpha_{1}} \ldots\left(\partial / \partial x_{n}\right)^{\alpha_{n}}, m=$ $1, \ldots, M_{k}$ and $k \in \mathbb{N}_{0}$.

Definition 3.1. Let $d \in(0, n]$ and $\Gamma$ be a compact $d$-set with a finite Radon measure $\mu$ in $\mathbb{R}^{n}$ as in Definition 1.1 . Let $\psi^{k, m}$ 's be the resolution of unity as above. Let $1<p \leq \infty$ and $s \geq 0$. Then

$$
(\beta q u)_{k m}(\gamma)=2^{-k(s-d / p)} 2^{k|\beta|}\left(\gamma-\gamma^{k, m}\right)^{\beta} \psi^{k, m}(\gamma)
$$

for $\gamma \in \Gamma$ with $\beta \in \mathbb{N}_{0}^{n}, k \in \mathbb{N}_{0}$ and $m=1, \ldots, M_{k}$, is called an $(s, p)$ - $\beta$-quark related to the ball $B_{k m}$, where $\gamma^{\beta}=\gamma_{1}^{\beta_{1}} \ldots \gamma_{n}^{\beta_{n}}$ for $\beta \in \mathbb{N}_{0}^{n}$.

In [16], Triebel introduced the Besov spaces $B_{p q}^{s}(\Gamma)$ via quarks as follows. First we recall that for $\lambda=\left\{\lambda_{k m} \in \mathbb{C}: k \in \mathbb{N}_{0}, m=1, \ldots, M_{k}\right\}$, we define the spaces $b_{p q}(\Gamma)$ by

$$
b_{p q}(\Gamma)=\left\{\lambda:\|\lambda\|_{b_{p q}(\Gamma)}=\left\{\sum_{k=0}^{\infty}\left(\sum_{m=1}^{M_{k}}\left|\lambda_{k m}\right|^{p}\right)^{q / p}\right\}^{1 / q}<\infty\right\} .
$$

Definition 3.2. Let $d \in(0, n]$ and $\Gamma$ be a compact $d$-set with a finite Radon measure $\mu$ in $\mathbb{R}^{n}$ as in Definition 1.1. Let $s>0,1<p<\infty$ and $0<q \leq \infty$. Let $(\beta q u)_{k m}$ be the $(s, p)$ - $\beta$-quarks on $\Gamma$ as in Definition 3.1. We put $\lambda=\left\{\lambda^{\beta}: \beta \in \mathbb{N}_{0}^{n}\right\}$ with

$$
\lambda^{\beta}=\left\{\lambda_{k m}^{\beta} \in \mathbb{C}: k \in \mathbb{N}_{0}, m=1, \ldots, M_{k}\right\} .
$$

Let $\sigma>r$ with $2^{r}=2 C_{2}$, where $C_{2}$ is as in (3.1), let $\lambda^{\beta} \in b_{p q}(\Gamma)$ and

$$
\|\lambda\|_{b_{p q}(\Gamma)}^{\sigma}=\sup _{\beta \in \mathbb{N}_{0}^{n}} 2^{\sigma|\beta|}\left\|\lambda^{\beta}\right\|_{b_{p q}(\Gamma)}<\infty .
$$

Then $B_{p q}^{s}(\Gamma)_{\sigma}$ is the collection of all $g \in L^{1}(\Gamma)$ which can be represented as

$$
g(\gamma)=\sum_{\beta \in \mathbb{N}_{0}^{n}} \sum_{k=0}^{\infty} \sum_{m=1}^{M_{k}} \lambda_{k m}^{\beta}(\beta q u)_{k m}(\gamma), \quad \gamma \in \Gamma
$$

with (3.2). Furthermore,

$$
\|g\|_{B_{p q}^{s}(\Gamma)_{\sigma}}=\inf \|\lambda\|_{b_{p q}(\Gamma)}^{\sigma}
$$

where the infimum is taken over all admissible representations.

The following lemma is a simple case of Theorem 9.33 in [16].

LEMma 3.1. Let $\Gamma$ be a compact $d$-set with a finite Radon measure $\mu$ in $\mathbb{R}^{n}$ as in Definition 1.1. Let $d \in(0, n], s>0,1<p<\infty$ and $0<q \leq \infty$. Then the spaces $B_{p q}^{s}(\Gamma)_{\sigma}$ introduced in Definition 3.2 are independent of the 
adopted resolutions of unity as above and of the number $\sigma$ (with equivalent quasi-norms), and will be denoted simply by $B_{p q}^{s}(\Gamma)$. Furthermore,

$$
B_{p q}^{s}(\Gamma)=\operatorname{tr}_{\Gamma} B_{p q}^{s+(n-d) / p}\left(\mathbb{R}^{n}\right)
$$

with equivalent quasi-norms.

The first systematic study of the spaces $B_{p q}^{s}(\Gamma)$ on $d$-sets is due to Jonsson and Wallin [13]. The spaces were defined with the help of first and higher differences and approximation by polynomials. A result similar to Lemma 3.1 was obtained; see Theorem 1 in [13, p. 141]. In other words, under the conditions of Lemma 3.1, the spaces $B_{p q}^{s}(\Gamma)$ defined as above by use of quarks are the same as those defined by Jonsson and Wallin in [13, Definition 2, p. 123] via the methods mentioned above; see [13] for the details.

Now, we will show that under the conditions of Lemma 3.1, the spaces $B_{p q}^{s}(\Gamma)$ with $1 \leq q \leq \infty$ defined above are the same as those defined in Section 2 by regarding $\Gamma$ as a space of homogeneous type.

Temporarily, denote the Besov spaces on $\Gamma$ defined in Section 2 by $\bar{B}_{p q}^{s}(\Gamma)$.

TheOREM 3.1. Let $\Gamma$ be a compact $d$-set with a finite Radon measure $\mu$ in $\mathbb{R}^{n}$ as in Definition 1.1. Let $d \in(0, n], 1>s>0,1<p<\infty$ and $1 \leq q \leq \infty$. Then the spaces $B_{p q}^{s}(\Gamma)$ introduced in Definition 3.2 are the same as $\bar{B}_{p q}^{s}(\Gamma)$ introduced in Definition 2.3, with equivalent norms.

Proof. Let us first prove that $B_{p q}^{s}(\Gamma) \subset \bar{B}_{p q}^{s}(\Gamma)$. We only consider $p, q \in$ $(1, \infty)$; the other cases are simpler. Let $g \in B_{p q}^{s}(\Gamma)_{\sigma}$ for some admissible $\sigma$. By Definition 3.2, we then have the quarkonial decomposition

$$
g(x)=\sum_{\beta \in \mathbb{N}_{0}^{n}} \sum_{\nu=0}^{\infty} \sum_{m=1}^{M_{\nu}} \lambda_{\nu m}^{\beta}(\beta q u)_{\nu m}(x)
$$

for $x \in \Gamma$, with the notation of Definition 3.2. We wish to use Definition 2.3 to verify that $f \in \bar{B}_{p q}^{s}(\Gamma)$. We first remark that since we have chosen the metric in $\Gamma$ to be just the restriction to $\Gamma$ of the usual Euclidean metric, it follows that $\theta$ in (1.2) equals 1 in this case. We claim that if $k, \nu \in \mathbb{N} \cup\{0\}$ and $k \leq \nu$, then for $x \in \Gamma$,

$$
\begin{aligned}
& \left|E_{k}\left((\beta q u)_{\nu m}\right)(x)\right| \\
& \quad \leq C 2^{-(\nu-k) d}\left(2 C_{2}\right)^{|\beta|} 2^{-\nu(s-d / p)}\left(1+2^{k}\left|x-\gamma^{\nu, m}\right|\right)^{-(d+1)},
\end{aligned}
$$

where $C_{2}$ is as in (3.1), and that if $k, \nu \in \mathbb{N} \cup\{0\}$ and $k>\nu$, then for $x \in \Gamma$,

$$
\begin{aligned}
& \left|E_{k}\left((\beta q u)_{\nu m}\right)(x)\right| \\
& \leq C 2^{-(k-\nu)}\left(2 C_{2}\right)^{|\beta|} 2^{-\nu(s-d / p)}\left(1+2^{\nu}\left|x-\gamma^{\nu, m}\right|\right)^{-(d+1)} .
\end{aligned}
$$


In fact, we have

$$
\begin{aligned}
\left|E_{k}\left((\beta q u)_{\nu m}\right)(x)\right| & =\left|\int_{\Gamma} E_{k}(x, y)(\beta q u)_{\nu m}(y) d \mu(y)\right| \chi_{\left\{x \in \Gamma:\left|x-\gamma^{\nu, m}\right| \leq C 2^{-k}\right\}}(x) \\
& \leq \int_{\Gamma}\left|E_{k}(x, y)(\beta q u)_{\nu m}(y)\right| d \mu(y) \chi_{\left\{x \in \Gamma:\left|x-\gamma^{\nu, m}\right| \leq C 2^{-k}\right\}}(x) \\
& \leq C 2^{-(\nu-k) d}\left(2 C_{2}\right)^{|\beta|} 2^{-\nu(s-d / p)} \chi_{\left\{x \in \Gamma:\left|x-\gamma^{\nu, m}\right| \leq C 2^{-k}\right\}}(x) \\
& \leq C 2^{-(\nu-k) d}\left(2 C_{2}\right)^{|\beta|} 2^{-\nu(s-d / p)}\left(1+2^{k}\left|x-\gamma^{\nu, m}\right|\right)^{-(d+1)}
\end{aligned}
$$

where $C$ is independent of $\nu, m, k$ and $x$. This is (3.3).

For (3.4), since $k>\nu \geq 0$, we have

$$
\int_{\Gamma} E_{k}(x, y) d \mu(y)=0 ;
$$

and therefore, by the mean value theorem,

$$
\begin{aligned}
\left|E_{k}\left((\beta q u)_{\nu m}\right)(x)\right|= & \left|\int_{\Gamma} E_{k}(x, y)(\beta q u)_{\nu m}(y) d \mu(y)\right| \chi_{\left\{x \in \Gamma:\left|x-\gamma^{\nu, m}\right| \leq C 2^{-\nu}\right\}}(x) \\
= & \left|\int_{\Gamma} E_{k}(x, y)\left[(\beta q u)_{\nu m}(y)-(\beta q u)_{\nu m}(x)\right] d \mu(y)\right| \\
& \times \chi_{\left\{x \in \Gamma:\left|x-\gamma^{\nu, m}\right| \leq C 2^{-\nu}\right\}}(x) \\
\leq & C 2^{-(k-\nu)}\left(2 C_{2}\right)^{|\beta|} 2^{-\nu(s-d / p)} \chi_{\left\{x \in \Gamma:\left|x-\gamma^{\nu, m}\right| \leq C 2^{-\nu}\right\}}(x) \\
\leq & C 2^{-(k-\nu)}\left(2 C_{2}\right)^{|\beta|} 2^{-\nu(s-d / p)}\left(1+2^{\nu}\left|x-\gamma^{\nu, m}\right|\right)^{-(d+1)}
\end{aligned}
$$

where $C$ is independent of $\nu, m, k$ and $x$, proving (3.4).

Now we write

$$
\begin{aligned}
\left\{\sum_{k=0}^{\infty}\left(2^{k s}\left\|E_{k}(g)\right\|_{L^{p}(\Gamma)}\right)^{q}\right\}^{1 / q} & \\
\leq & \left\{\sum_{k=0}^{\infty}\left[2^{k s}\left\|E_{k}\left(\sum_{\beta \in \mathbb{N}_{0}^{n}} \sum_{\nu=0}^{k} \sum_{m=1}^{M_{\nu}} \lambda_{\nu m}^{\beta}(\beta q u)_{\nu m}\right)\right\|_{L^{p}(\Gamma)}\right]^{q}\right\}^{1 / q} \\
& +\left\{\sum_{k=0}^{\infty}\left[2^{k s}\left\|E_{k}\left(\sum_{\beta \in \mathbb{N}_{0}^{n}} \sum_{\nu=k+1}^{\infty} \sum_{m=1}^{M_{\nu}} \lambda_{\nu m}^{\beta}(\beta q u)_{\nu m}\right)\right\|_{L^{p}(\Gamma)}\right]^{q}\right\}^{1 / q} \\
= & I+I I .
\end{aligned}
$$

For $k \in \mathbb{N} \cup\{0\}, m=1, \ldots, M_{k}$, let

$$
\bar{B}_{k m}=\left\{x \in \Gamma:\left|x-\gamma^{k, m}\right| \leq C_{4} 2^{-k}\right\}
$$

and $C_{4} \leq C_{2}$, where $C_{2}$ and $\gamma^{k, m}$ 's are as in (3.1). Obviously, by suitably 
choosing $C_{4}$, we may suppose that

$$
\left\{\bar{B}_{k m}: k \in \mathbb{N} \cup\{0\}, m=1, \ldots, M_{k}\right\}
$$

satisfy the conditions on $\left\{Q_{\alpha}^{k} \subset X: k \in \mathbb{N} \cup\{0\}, \alpha \in M_{k}\right\}$ as in Lemma 2.1 for the present case. In what follows, for $q \in[1, \infty]$, we define $q^{\prime} \in[1, \infty]$ by $1 / q+1 / q^{\prime}=1$. Hence, by (3.4), (2.1) of Lemma 2.4, and Hölder's inequality, we have

$$
\begin{aligned}
I \leq & C\left\{\sum _ { k = 0 } ^ { \infty } \left[2^{k s} 2^{-\nu d / p}\right.\right. \\
& \left.\left.\times\left(\sum_{\beta \in \mathbb{N}_{0}^{n}} \sum_{\nu=0}^{k} \sum_{m=1}^{M_{\nu}}\left|\lambda_{\nu m}^{\beta}\right|^{p} 2^{-(k-\nu) p-\nu(s-d / p) p}\left(2 C_{2}\right)^{|\beta| p}\right)^{1 / p}\right]^{q}\right\}^{1 / q} \\
= & C\left\{\sum_{k=0}^{\infty}\left[\sum_{\beta \in \mathbb{N}_{0}^{n}} \sum_{\nu=0}^{k} \sum_{m=1}^{M_{\nu}}\left|\lambda_{\nu m}^{\beta}\right|^{p} 2^{-(k-\nu)(1-s) p}\left(2 C_{2}\right)^{|\beta| p}\right]^{q / p}\right\}^{1 / q} \\
\leq & C \sum_{\beta \in \mathbb{N}_{0}^{n}}\left(2 C_{2}\right)^{|\beta|}\left\{\sum_{k=0}^{\infty}\left[\sum_{\nu=0}^{k}\left(\sum_{m=1}^{M_{\nu}}\left|\lambda_{\nu m}^{\beta}\right|^{p}\right)^{q / p} 2^{-(k-\nu)(1-s) q / 2}\right]\right. \\
& \left.\times\left[\sum_{\nu=0}^{k} 2^{-(k-\nu)(1-s) q^{\prime} / 2}\right]^{q / q^{\prime}}\right\}^{1 / q} \\
\leq & C \sum_{\beta \in \mathbb{N}_{0}^{n}}\left(2 C_{2}\right)^{|\beta|}\left\|\lambda^{\beta}\right\|_{b_{p q}(\Gamma)} \leq C\|g\|_{B_{p q}^{s}(\Gamma)} \sum_{\beta \in \mathbb{N}_{0}^{n}} 2^{(r-\sigma)|\beta|} \leq C\|g\|_{B_{p q}^{s}(\Gamma)},
\end{aligned}
$$

which is the desired estimate for $I$; we used the fact that $s<1$.

In what follows, let $M$ be the Hardy-Littlewood maximal operator on $\Gamma$. It is well known that $M$ is bounded in $L^{p}(\Gamma)$ for $p \in(1, \infty)$; see [2], or Theorem 2.2 in [12], or Theorem 14.13 in [6]. Using this for $I I$, by (3.3), (2.2) of Lemma 2.4 and Hölder's inequality, we have

$$
\begin{aligned}
I I \leq & C \sum_{\beta \in \mathbb{N}_{0}^{n}}\left\{\sum_{k=0}^{\infty}\left[\sum_{\nu=k+1}^{\infty} 2^{k s}\left\|\sum_{m=1}^{M_{\nu}} \lambda_{\nu m}^{\beta} E_{k}\left[(\beta q u)_{\nu m}\right]\right\|_{L^{p}(\Gamma)}\right]^{q}\right\}^{1 / q} \\
\leq & C \sum_{\beta \in \mathbb{N}_{0}^{n}}\left(2 C_{2}\right)^{|\beta|} \\
& \times\left\{\sum_{k=0}^{\infty}\left[\sum_{\nu=k+1}^{\infty} 2^{-(\nu-k) s} 2^{\nu d / p}\left\|M\left(\sum_{m=1}^{M_{\nu}}\left|\lambda_{\nu m}^{\beta}\right| \chi_{\bar{B}_{\nu m}}\right)\right\|_{L^{p}(\Gamma)}\right]^{q}\right\}^{1 / q} \\
\leq & C \sum_{\beta \in \mathbb{N}_{0}^{n}}\left(2 C_{2}\right)^{|\beta|}\left\{\sum_{k=0}^{\infty}\left[\sum_{\nu=k+1}^{\infty} 2^{-(\nu-k) s} 2^{\nu d / p}\left\|\sum_{m=1}^{M_{\nu}}\left|\lambda_{\nu m}^{\beta}\right| \chi_{\bar{B}_{\nu m}}\right\|_{L^{p}(\Gamma)}\right]^{q}\right\}^{1 / q}
\end{aligned}
$$




$$
\begin{aligned}
& \leq C \sum_{\beta \in \mathbb{N}_{0}^{n}}\left(2 C_{2}\right)^{|\beta|}\left\{\sum_{k=0}^{\infty}\left[\sum_{\nu=k+1}^{\infty} 2^{-(\nu-k) s}\left(\sum_{m=1}^{M_{\nu}}\left|\lambda_{\nu m}^{\beta}\right|^{p}\right)^{1 / p}\right]^{q}\right\}^{1 / q} \\
& \leq C \sum_{\beta \in \mathbb{N}_{0}^{n}}\left(2 C_{2}\right)^{|\beta|}\left\{\sum_{k=0}^{\infty}\left[\sum_{\nu=k+1}^{\infty} 2^{-(\nu-k) s q / 2}\left(\sum_{m=1}^{M_{\nu}}\left|\lambda_{\nu m}^{\beta}\right|^{p}\right)^{q / p}\right]\right. \\
& \left.\times\left[\sum_{\nu=k+1}^{\infty} 2^{-(\nu-k) s q^{\prime} / 2}\right]^{q / q^{\prime}}\right\}^{1 / q} \\
& \leq C \sum_{\beta \in \mathbb{N}_{0}^{n}}\left(2 C_{2}\right)^{|\beta|}\left\|\lambda^{\beta}\right\|_{b_{p q}(\Gamma)} \leq C\|g\|_{B_{p q}^{s}(\Gamma)} \sum_{\beta \in \mathbb{N}_{0}^{n}} 2^{(r-\sigma)|\beta|} \leq C\|g\|_{B_{p q}^{s}(\Gamma)},
\end{aligned}
$$

which is the desired estimate for $I I$; we used the fact that $s>0$ and in the fourth step, we used the property satisfied by the balls

$$
\left\{\bar{B}_{\nu m}: \nu \in \mathbb{N} \cup\{0\}, m=1, \ldots, M_{\nu}\right\} .
$$

This proves that $B_{p q}^{s}(\Gamma) \subset \bar{B}_{p q}^{s}(\Gamma)$.

Now, let us prove $\bar{B}_{p q}^{s}(\Gamma) \subset B_{p q}^{s}(\Gamma)$. To do that, we need first to show that our 1-smooth blocks and 1-smooth atoms in Lemma 2.2 belong to $C^{\infty}\left(\mathbb{R}^{n}\right)$. For this purpose, by the proof of Theorem 2.1 in [8], we only need to prove that in the present case, we can choose an approximation to the identity as in Definition 2.2 with $C^{\infty}\left(\mathbb{R}^{n} \times \mathbb{R}^{n}\right)$ kernels. This can be done by using Coifman's idea as follows (see [3]). We choose a nonnegative radial $C^{\infty}\left(\mathbb{R}^{n}\right)$ function $g$ with $g(x)=0$ if $|x|>2, g(x)=1$ if $|x| \leq 1$ and $0 \leq g(x) \leq 1$ for all $x \in \mathbb{R}^{n}$. We then define the operator $T_{k}$ by

$$
T_{k}(f)(x)=\int_{\Gamma} 2^{k d} g\left(2^{k}(x-y)\right) f(y) d \mu(y),
$$

where $k \in \mathbb{N} \cup\{0\}$. It is easy to check that there are two constants $C$ and $C^{\prime}$ such that

$$
C^{\prime} \leq T_{k}(1)(x) \leq C
$$

for all $x \in \Gamma$. In fact, it is easy to see that for some small $\varepsilon_{k}>0$, $C \leq T_{k}(1)(x)$ when $x \in \Gamma_{\varepsilon_{k}}$. For example, we can take $\varepsilon_{k}=2^{-(k+1)}$. We then choose a Schwartz function $\psi_{k} \in C^{\infty}\left(\mathbb{R}^{n}\right)$ such that $\psi_{k}(x)=1$ for $x \in \Gamma, \psi_{k}(x)>0$ for $x \in \Gamma_{\varepsilon_{k} / 3}$ and $\psi_{k}(x)=0$ for $x \notin \Gamma_{\varepsilon_{k} / 2}$. Moreover, $0 \leq \psi_{k}(x) \leq 1$ for all $x \in \mathbb{R}^{n}$. We now define the operators $M_{k}$ and $W_{k}$ as follows:

$$
M_{k}(f)(x)=\frac{\psi_{k}(x)}{T_{k}(1)(x)} f(x),
$$

so $M_{k}$ is just the multiplication by $\psi_{k}(x) / T_{k}(1)(x)$, and

$$
W_{k}(f)(x)=\frac{1}{T_{k}\left[\frac{1}{T_{k}(1)(\cdot)}\right](x)} f(x),
$$


again a multiplication operator; here $f$ is any suitable function on $\Gamma$. Then the operator $\left\{S_{k}\right\}_{k \in \mathbb{N} \cup\{0\}}$ defined by

$$
S_{k}(f)=M_{k} T_{k} W_{k} T_{k} M_{k}(f)
$$

is the approximation to the identity we are looking for.

Let $f \in \bar{B}_{p q}^{s}(\Gamma)$. By Lemma 2.2, there exist a sequence

$$
\lambda=\left\{\lambda_{Q_{\tau}^{k}}: k \in \mathbb{N} \cup\{0\}, \tau=1, \ldots, M_{k}\right\} \in b_{p q}^{s}(\Gamma),
$$

1-smooth atoms $\left\{a_{Q_{\tau}^{k}}: k \in \mathbb{N}, \tau=1, \ldots, M_{k}\right\}$ and 1-smooth blocks $\left\{a_{Q_{\tau}^{0}}\right.$ : $\left.\tau=1, \ldots, M_{0}\right\}$ such that

$$
f=\sum_{k=0}^{\infty} \sum_{\tau=1}^{M_{k}} \lambda_{Q_{\tau}^{k}} a_{Q_{\tau}^{k}}
$$

with convergence in $\bar{B}_{p q}^{s}(\Gamma)$ and

$$
\|\lambda\|_{b_{p q}^{s}(\Gamma)} \leq C\|f\|_{\bar{B}_{p q}^{s}(\Gamma)}
$$

where $b_{p q}^{s}(\Gamma)$ is the collection of sequences

$$
\lambda=\left\{\lambda_{Q_{\tau}^{k}}: k \in \mathbb{N} \cup\{0\}, \tau=1, \ldots, M_{k}\right\}
$$

such that

$$
\|\lambda\|_{b_{p q}^{s}(\Gamma)}=\left\{\sum_{k=0}^{\infty}\left[\sum_{\tau=1}^{M_{k}}\left(\mu\left(Q_{\tau}^{k}\right)^{-s / d-1 / 2+1 / p}\left|\lambda_{Q_{\tau}^{k}}\right|\right)^{p}\right]^{q / p}\right\}^{1 / q}
$$

is finite. Moreover, by the above construction of the $C^{\infty}$ approximation to the identity and the proof of Theorem 2.1 in [8], we know that $\left\{a_{Q_{\tau}^{k}}\right.$ : $\left.k \in \mathbb{N}, \tau=1, \ldots, M_{k}\right\}$ and $\left\{a_{Q_{\tau}^{0}}: \tau=1, \ldots, M_{0}\right\}$ belong to $C^{\infty}\left(\mathbb{R}^{n}\right)$. Note that Lemma 2.2 is also true when $X=\mathbb{R}^{n}, d=n$ and $\theta=1$. In this case, we choose $\mu=m$, the $n$-dimensional Lebesgue measure on $\mathbb{R}^{n}$. In fact, to remove the restriction on $s$, we can just use the results in [4]. When $|s|<1$, the spaces obtained by Lemma 2.2 and those in [4] are the same. Let us denote temporarily the Besov spaces on $\mathbb{R}^{n}$ by $\widetilde{B}_{p q}^{s}\left(\mathbb{R}^{n}\right)$. As we have just pointed out, $a_{Q_{\tau}^{k}}$ for all $k \in \mathbb{N} \cup\{0\}$ and $\tau=1, \ldots, M_{k}$ can be automatically extended to $\mathbb{R}^{n}$. We can also suppose that $\left\{Q_{\tau}^{k}: k \in \mathbb{N} \cup\{0\}, \tau=1, \ldots, M_{k}\right\}$ of Lemma 2.1 for $\Gamma$ are just the restrictions to $\Gamma$ of $\left\{\widetilde{Q}_{\tau}^{k}: k \in \mathbb{N} \cup\{0\}, \tau=1, \ldots, \widetilde{M}_{k}\right\}$ from Lemma 2.1 for $\mathbb{R}^{n}$, which are just the dyadic cubes in $\mathbb{R}^{n}$; see [4]. Thus, if $Q_{\tau}^{k}=\widetilde{Q}_{\tau}^{k} \cap \Gamma$, we define $a_{\widetilde{Q}_{\tau}^{k}}=C_{0} 2^{k(n-d) / 2} a_{Q_{\tau}^{k}}$, and $a_{\widetilde{Q}_{\tau}^{k}}=0$ otherwise. Then we have

$$
\begin{gathered}
\operatorname{supp} a_{\widetilde{Q}_{\tau}^{k}} \subset 3 \widetilde{Q}_{\tau}^{k}=\left\{x \in \mathbb{R}^{n}:\left|x-z_{\tau}^{k}\right|<3 C 2^{-k}\right\}, \\
\left|a_{\widetilde{Q}_{\tau}^{k}}(x)\right| \leq C_{0} C_{0}^{\prime} 2^{k(n-d) / 2} 2^{k d / 2}=m\left(\widetilde{Q}_{\tau}^{k}\right)^{-1 / 2}, \\
\left|a_{\widetilde{Q}_{\tau}^{k}}(x)-a_{\widetilde{Q}_{\tau}^{k}}(y)\right| \leq C_{0} C_{0}^{\prime}|x-y| 2^{k(n-d) / 2} 2^{k d(1 / 2+1 / d)}=m\left(\widetilde{Q}_{\tau}^{k}\right)^{-1 / 2-1 / n}|x-y|,
\end{gathered}
$$


by choosing a suitable constant $C_{0}$ which is independent of $x, y, k$ and $\tau$, where $C_{0}^{\prime}$ depends only on $d$ and $\mu$. Thus,

$$
\left\{a_{\widetilde{Q}_{\tau}^{k}}: k \in \mathbb{N} \cup\{0\}, \tau=1, \ldots, \widetilde{M}_{k}\right\}
$$

are 1-smooth blocks. Moreover, let $\lambda_{\widetilde{Q}_{\tau}^{k}}=C_{0}^{-1} 2^{k(d-n) / 2} \lambda_{Q_{\tau}^{k}}$ if $Q_{\tau}^{k}=\widetilde{Q}_{\tau}^{k} \cap \Gamma$, and $\lambda_{\widetilde{Q}_{\tau}^{k}}=0$ otherwise. Let $\widetilde{\lambda}=\left\{\lambda_{\widetilde{Q}_{\tau}^{k}}: k \in \mathbb{N} \cup\{0\}, \tau=1, \ldots, \widetilde{M}_{k}\right\}$. Then

$$
\begin{aligned}
\|\widetilde{\lambda}\|_{b_{p q}^{s+(n-d) / p}\left(\mathbb{R}^{n}\right)} & \leq C\left\{\sum_{k=0}^{\infty}\left[\sum_{\tau=1}^{\widetilde{M}_{k}}\left(2^{k(s+(n-d) / p)+k n / 2-k n / p}\left|\lambda_{\widetilde{Q}_{\tau}^{k}}\right|\right)^{p}\right]^{q / p}\right\}^{1 / q} \\
& =C\left\{\sum_{k=0}^{\infty}\left[\sum_{\tau=1}^{\widetilde{M}_{k}}\left(2^{k s+k d / 2-k d / p}\left|\lambda_{Q_{\tau}^{k}}\right|\right)^{p}\right]^{q / p}\right\}^{1 / q} \\
& =C\|\lambda\|_{b_{p q}^{s}(\Gamma)}<\infty
\end{aligned}
$$

where $C$ is independent of $f$. Now, if we define

$$
\tilde{f}=\sum_{k=0}^{\infty} \sum_{\tau=1}^{\widetilde{M}_{k}} \lambda_{\widetilde{Q}_{\tau}^{k}} a_{\widetilde{Q}_{\tau}^{k}},
$$

then by noting that $0<s<1$ and using Lemma 2.3 (see also the proof of Theorem 2.1 in [8]), we find that $\tilde{f} \in \widetilde{B}_{p q}^{s+(n-d) / p}\left(\mathbb{R}^{n}\right), f=\operatorname{tr}_{\Gamma} \tilde{f}$ and

$$
\|\widetilde{f}\|_{\widetilde{B}_{p q}^{s+(n-d) / p}\left(\mathbb{R}^{n}\right)} \leq C\|f\|_{\bar{B}_{p q}^{s}(\Gamma)},
$$

where $C$ is independent of $f$. Thus, by Lemma 3.1, $f \in B_{p q}^{s}(\Gamma)$, and

$$
\|f\|_{B_{p q}^{s}(\Gamma)} \leq C\|f\|_{\bar{B}_{p q}^{s}(\Gamma)},
$$

where $C$ is independent of $f$.

This finishes the proof of Theorem 3.1.

The following corollary has been proved in the course of proof of Theorem 3.1 .

Corollary 3.1. Let $d \in(0, n]$ and let $\Gamma$ be a d-set with a Radon measure $\mu$ in $\mathbb{R}^{n}$ as in Definition 1.1. Then there is a $C^{\infty}$ approximation $\left\{S_{k}\right\}_{k=0}^{\infty}$ to the identity on $\Gamma$ in the following sense: for all $k \geq 0$ and all $x, y \in \Gamma$, the kernel $S_{k}(x, y)$ of $S_{k}$ is a $C^{\infty}$ function from $\mathbb{R}^{n} \times \mathbb{R}^{n}$ into $\mathbb{C}$ satisfying

(i) $S_{k}(x, y)=0$ if $|x-y| \geq C 2^{-k}, x, y \in \mathbb{R}^{n}$;

(ii) $\left|D_{x}^{\alpha} D_{y}^{\beta} S_{k}(x, y)\right| \leq C_{\alpha, \beta} 2^{k(d+|\alpha|+|\beta|)}, \alpha, \beta \in \mathbb{N}_{0}^{n}, x, y \in \mathbb{R}^{n}$;

(iii) $\int_{\Gamma} S_{k}(x, y) d \mu(y)=1$ for all $x \in \Gamma$;

(iv) $\int_{\Gamma} S_{k}(x, y) d \mu(x)=1$ for all $y \in \Gamma$, 
where $D_{x}^{\alpha}$ and $D_{y}^{\beta}$ are differential operators in $\mathbb{R}^{n}$ defined as above, with respect to $x$ and $y$, and $C$ and $C_{\alpha, \beta}$ are constants independent of $k, x$ and $y$.

\section{References}

[1] M. Christ, $A T(b)$ theorem with remarks on analytic capacity and the Cauchy integral, Colloq. Math. 60/61 (1990), 601-628.

[2] R. R. Coifman et G. Weiss, Analyse Harmonique Non-commutative sur Certains Espaces Homogènes, Lecture Notes in Math. 242, Springer, Berlin, 1971.

[3] G. David, J.-L. Journé et S. Semmes, Opérateurs de Calderón-Zygmund, fonctions para-accretives et interpolation, Rev. Mat. Iberoamericana 1 (1985), 1-56.

[4] M. Frazier and B. Jawerth, Decomposition of Besov spaces, Indiana Univ. Math. J. 34 (1985), 777-799.

[5] -, 一, A discrete transform and decompositions of distribution spaces, J. Funct. Anal. 93 (1990), 34-170.

[6] P. Hajłasz and P. Koskela, Sobolev met Poincaré, Mem. Amer. Math. Soc. 688 (2000).

[7] Y. S. Han, Inhomogeneous Calderón reproducing formula on spaces of homogeneous type, J. Geom. Anal. 7 (1997), 259-284.

[8] Y. S. Han, S. Lu and D. C. Yang, Inhomogeneous Besov and Triebel-Lizorkin spaces on spaces of homogeneous type, Approx. Theory Appl. 15 (1999), 37-65.

[9] - , - - Inhomogeneous Triebel-Lizorkin spaces on spaces of homogeneous type, Math. Sci. Res. Hot-Line 3 (1999), no. 9, 1-29.

[10] Y. S. Han and E. T. Sawyer, Littlewood-Paley theory on spaces of homogeneous type and classical function spaces, Mem. Amer. Math. Soc. 530 (1994).

[11] Y. S. Han and D. C. Yang, New characterizations and applications of inhomogeneous Besov and Triebel-Lizorkin spaces on homogeneous type spaces and fractals, Dissertationes Math. (Rozprawy Mat.) 403 (2002).

[12] J. Heinonen, Lectures on Analysis on Metric Spaces, Springer, Berlin, 2001.

[13] A. Jonsson and H. Wallin, Function Spaces on Subsets of $\mathbb{R}^{n}$, Math. Reports 2, Harwood, London, 1984.

[14] R. A. Macías and C. Segovia, Lipschitz functions on spaces of homogeneous type, Adv. Math. 33 (1979), 257-270.

[15] H. Triebel, Fractals and Spectra, Birkhäuser, Basel, 1997.

[16] —, The Structure of Functions, Birkhäuser, Basel, 2001.

Department of Mathematics

Beijing Normal University

Beijing 100875

People's Republic of China

E-mail: dcyang@bnu.edu.cn

Received August 9, 2001

Revised version October 18, 2002 